\title{
Molecular Orbital Study on Photoreaction of Peroxy Radicals of Polytetrafluoroethylene
}

\author{
Akihiro NoguchI, Shin-ichi Kondo, and Masayuki KuzuYA* \\ Laboratory of Pharmaceutical Physical Chemistry, Gifu Pharmaceutical University, 5-6-1, Mitahora-higashi, Gifu \\ 502, Japan. Received June 23, 1993
}

The photoreaction of peroxy radicals of polytetrafluoroethylene (PTFE) was investigated by means of the molecular orbital (intermediate neglect of differential overlap/spectroscopy-configuration interaction (INDO/S-CI)) calculations of the excited states of model compounds for two types of radicals, i.e. the endchain peroxy and midchain peroxy radicals. Calculations showed that the midchain radicals are excited to the second lowest excited doublet $\left(\mathrm{D}_{2}\right)$ state, which is responsible for the photoreaction, with much higher efficiency than the endchain peroxy radicals. The bond order perturbations accompanied by the transition from the ground doublet $\left(D_{0}\right)$ state to the $D_{2}$ state in the midchain radicals seem to well explain the experimental results.

Keywords polytetrafluoroethylene; peroxy radical; photoreaction; molecular orbital; intermediate neglect of differential overlap/spectroscopy-configuration interaction (INDO/S-CI)

In previous papers we have reported the nature of radicals formed by plasma irradiation of polytetrafluoroethylene (PTFE) and of the peroxy radicals formed on exposure to air. ${ }^{1,2)}$ That is, argon plasma irradiation of PTFE gave midchain radicals as a major product, endchain radicals formed by cleavage of the main chain and immobilized dangling-bond sites in the cross-linked part near the polymer surface. These radicals were immediately oxidized on exposure to air to give the corresponding peroxy radicals. The midchain peroxy radical was easily decomposed by UV (ultraviolet) irradiation to give endchain perfluoroalkyl radicals, while the endchain peroxy radical is known to be inactive with UV irradiation. ${ }^{3,4}$ ) The photolabile property of the peroxy radical of PTFE is quite interesting, in contrast with the high thermal and photochemical stabilities of PTFE.

With a view to gaining insight into the nature of the electronic structure of peroxy radicals of PTFE in the excited state, we have carried out molecular orbital (MO) calculations of the excited states for the model compounds of the endchain peroxy and midchain peroxy radicals using the INDO/S-CI (intermediate neglect of differential overlap/spectroscopy-configuration interaction) method. ${ }^{5)}$ Calculations were also performed for the model compounds of peroxy radicals of polyethylene.

\section{Results and Discussion}

We used perfluoro pentyldioxy radical (1) as a model compound of endchain peroxy radicals of PTFE and perfluoro 1-ethylpropyldioxy radical (2) as that of midchain peroxy radicals. Pentyldioxy radical (3) and 1ethylpropyldioxy radical (4) were used as model compounds of peroxy radicals of polyethylene. The calculated excitation energies and the oscillator strengths for the examined peroxy radicals are shown in Table $I$.

The calculated transition energies from the ground doublet state $\left(D_{0}\right)$ to the lowest excited doublet $\left(D_{1}\right)$ state were quite low ( $c a .0 .5 \mathrm{eV}$ ) for all the peroxy radicals and those from the $\mathrm{D}_{0}$ states to the second lowest excites doublet $\left(\mathrm{D}_{2}\right)$ states were in the region of $c a .5-6 \mathrm{eV}$. Taking account of the reported experimental conditions and the results of photoreaction for peroxy radicals of PTFE, ${ }^{3,4)}$ the photoreactions are apparently associated with excitation by a light from the UV region. Thus, the $\mathrm{D}_{1}$ states seem not to be responsible for the photoreaction and the excited states that are associated with the photoreactions are considered to be the $\mathrm{D}_{2}$ states. Also, in comparison of the $D_{0} \rightarrow D_{2}$ transition in 1 with that in 2, the calculated transition energy $(6.10 \mathrm{eV})$ in 1 was higher than that in $\mathbf{2}$ and was also higher than the applied light energy (below ca. $5 \mathrm{eV}$ ) in the experiments; also, the calculated oscillator strength in 1 was smaller than that in 2. So, it is considered that midchain peroxy radicals of PTFE are excited to the $\mathrm{D}_{2}$ state with much higher efficiency than the endchain radicals with UV irradiation. On the other hand, such a large difference in the property of transition between the endchain peroxy and midchain peroxy radicals was not shown for alkyl peroxy radicals.

Next, the photoreactions of peroxy radicals were investigated based on the electronic properties of the excited states. The configuration interaction $(\mathrm{CI})$ features of the $D_{2}$ and $D_{1}$ states in $\mathbf{1}-\mathbf{4}$ are shown in Figs. $1-4$, together with the coefficient distributions and nodal properties of the relevant MO's.

The CI features of the $\mathrm{D}_{1}$ states were nearly identical with one another for all the examined peroxy radicals and were mainly composed of the transitions from the highest occupied MO's (HOMO's) in the ground state to the singly occupied MO's (SOMO's), both of which are $\pi$-type antibonding orbitals localized on the peroxy groups. On the other hand, the CI feature of the $D_{2}$ state in the

TABLE I. Calculated Transition Energies $(\Delta E$ in $\mathrm{eV})$ and Oscillator Strengths $(f)$

\begin{tabular}{|c|c|c|c|c|c|c|c|c|c|}
\hline \multirow{2}{*}{\multicolumn{2}{|c|}{ Transition }} & \multicolumn{2}{|c|}{1} & \multicolumn{2}{|c|}{2} & \multicolumn{2}{|c|}{3} & \multicolumn{2}{|c|}{4} \\
\hline & & $\Delta E$ & $f$ & $\Delta E$ & $f$ & $\Delta E$ & $f$ & $\Delta E$ & $f$ \\
\hline $\mathrm{D}_{0}$ & $\mathrm{D}_{1}$ & 0.51 & 0.000 & 0.57 & 0.000 & 0.77 & 0.000 & 0.75 & 0.000 \\
\hline $\mathrm{D}_{0}$ & $\mathrm{D}_{2}$ & 6.10 & 0.002 & 4.92 & 0.145 & 5.39 & 0.162 & 4.91 & 0.156 \\
\hline $\mathrm{D}_{0}$ & $D_{3}$ & 6.29 & 0.005 & 5.72 & 0.001 & 5.92 & 0.001 & 5.37 & 0.002 \\
\hline
\end{tabular}




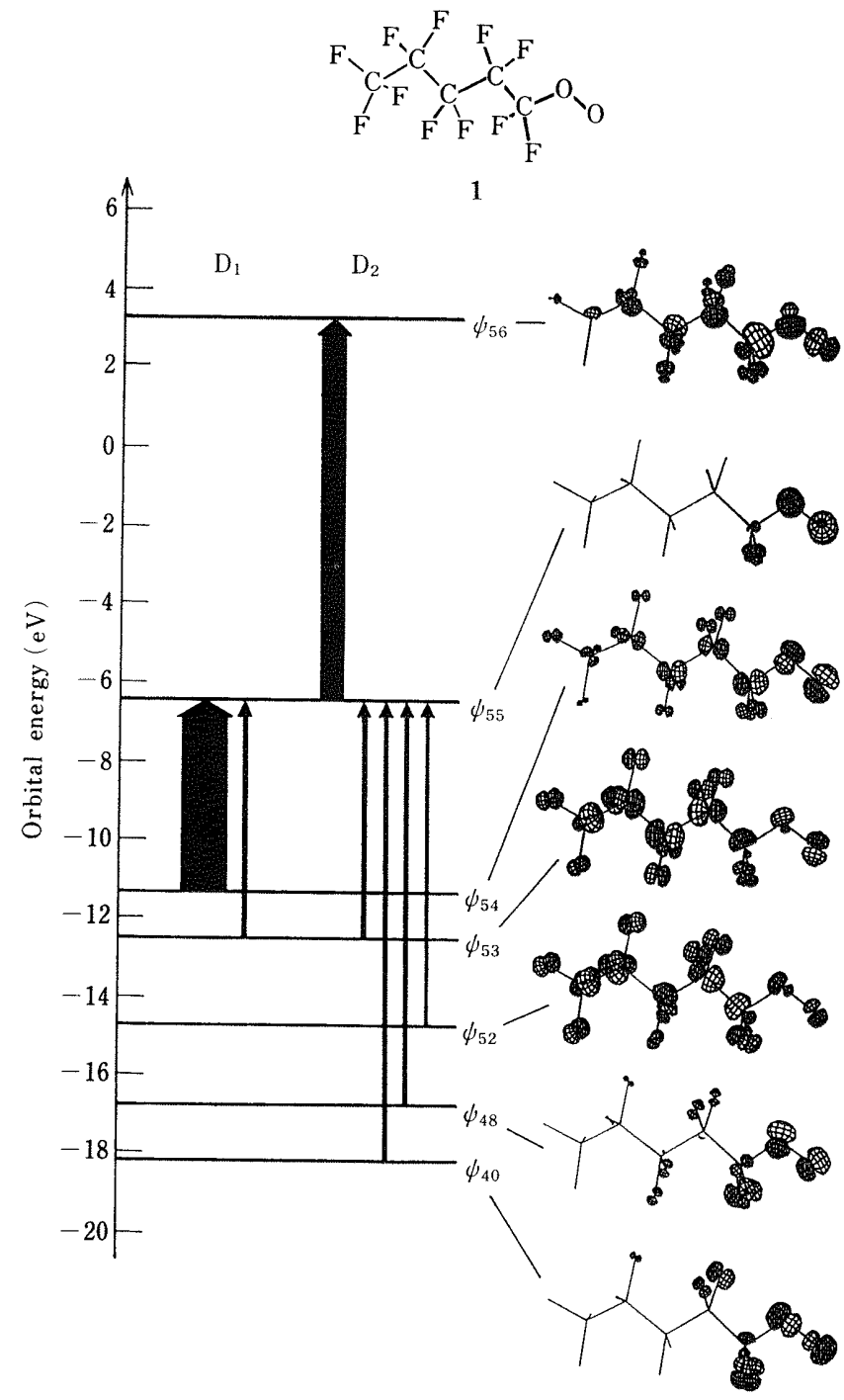

Fig. 1. Configuration Mixing in the $\mathrm{D}_{1}$ and $\mathrm{D}_{2}$ States and the Relevant Molecular Orbitals for 1

endchain perfluoro alkyldioxy radical, 1, was apparently different from that in the midchain peroxy radical, 2. That is, the $\mathrm{D}_{2}$ state in 1 mainly consisted of the transition from the SOMO $\left(\psi_{55}\right)$ to the lowest unoccupied MO (LUMO, $\psi_{56}$ ) which is an antibonding $\sigma$-orbital localized on the $\mathrm{C}-\mathrm{O}-\mathrm{O}$ moiety. The $\mathrm{D}_{2}$ state in 2 mainly consisted of the transition from the doubly occupied bonding $\mathrm{C}-\mathrm{C}$ $\sigma$-orbital $\left(\psi_{53}\right)$ to the SOMO $\left(\psi_{55}\right)$. For alkyldioxy radicals, the $\mathrm{D}_{2}$ state in 3 mainly consisted of two transitions from the bonding $\mathrm{C}-\mathrm{H} \sigma$-orbitals $\left(\psi_{15}\right.$ and $\left.\psi_{18}\right)$ to the $\operatorname{SOMO}\left(\psi_{22}\right)$. And, the $\mathrm{D}_{2}$ state in 4 mainly consisted of the transition from the bonding $\mathrm{C}-\mathrm{C} \sigma$-orbital $\left(\psi_{20}\right)$ to the SOMO $\left(\psi_{22}\right)$ and was similar to that in 2.

It was shown that for both perfluoro alkyl and alkyl compounds the $\mathrm{CI}$ features of the $\mathrm{D}_{2}$ state in the endchain peroxy radicals differ from those in the midchain peroxy radicals. Also, in the endchain peroxy radicals, the relevant MO's involved in the $\mathrm{D}_{2}$ state of perfluoro alkyldioxy radicals apparently differ from those of alkyldioxy radicals, but in the midchain peroxy radicals those of perfluoro alkyldioxy are similar.<smiles>OC(F)(C(F)(F)F)C(F)(F)C(F)(F)C(F)(F)F</smiles>

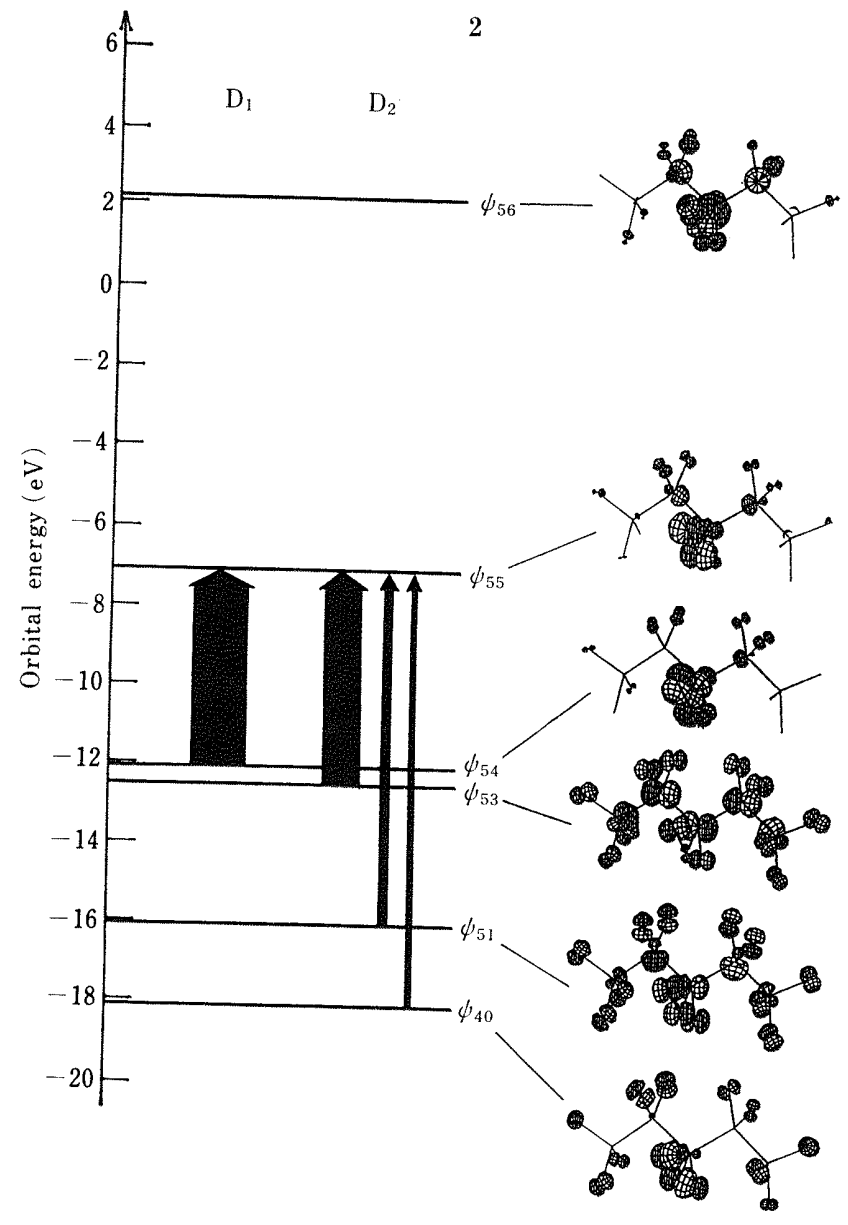

Fig. 2. Configuration Mixing in the $\mathrm{D}_{1}$ and $\mathrm{D}_{2}$ States and the Relevant Molecular Orbitals for 2

The photoreactions of peroxy radicals were investigated based on the perturbation $(\Delta P)$ in the bond order (atomic bond population) accompanied by the $\mathrm{D}_{0} \rightarrow \mathrm{D}_{2}$ transition. The $\Delta P$ values were calculated according to the following equation:

$$
\Delta P_{\mathrm{AB}}=\sum_{\mu}^{\text {onA onB }} \sum_{v}^{2} d_{k,(i-j)}^{2}\left(C_{\mu}^{j} C_{v}^{j}-C_{\mu}^{i} C_{v}^{i}\right) S_{\mu v}
$$

where $\mu$ and $v$ represent atomic orbitals on atoms $\mathrm{A}$ and $\mathrm{B}$, respectively, $d_{k,(i-j)}$ is the CI coefficient of $k$ th electronic configuration formed by the transition of one electron from $i$ th $\mathrm{MO}$ to $j$ th $\mathrm{MO}, C_{\mu}^{j}$ means the coefficient of atomic orbital $\mu$ in $j$ th $\mathrm{MO}$, and $S_{\mu \nu}$ is the overlap integral between atomic orbitals $\mu$ and $v$.

The results are shown in Fig. 5. For perfluoro alkyldioxy radicals, the $D_{0} \rightarrow D_{2}$ transition in 1 showed remarkable decreases in the $\mathrm{C}-\mathrm{O}$ and $\mathrm{O}-\mathrm{O}$ bond orders, and that in 2 showed a marked decrease in the $\mathrm{O}-\mathrm{O}$ bond order and noticeable decrease in the $\beta-\mathrm{C}-\mathrm{C}$ bond order. For alkyldioxy radicals, the features of the $\Delta P$ values in 4 were similar to those in 2 , but those in 3 were different from those in $\mathbf{1}$ and showed a marked decrease in the $\mathrm{O}-\mathrm{O}$ bond 


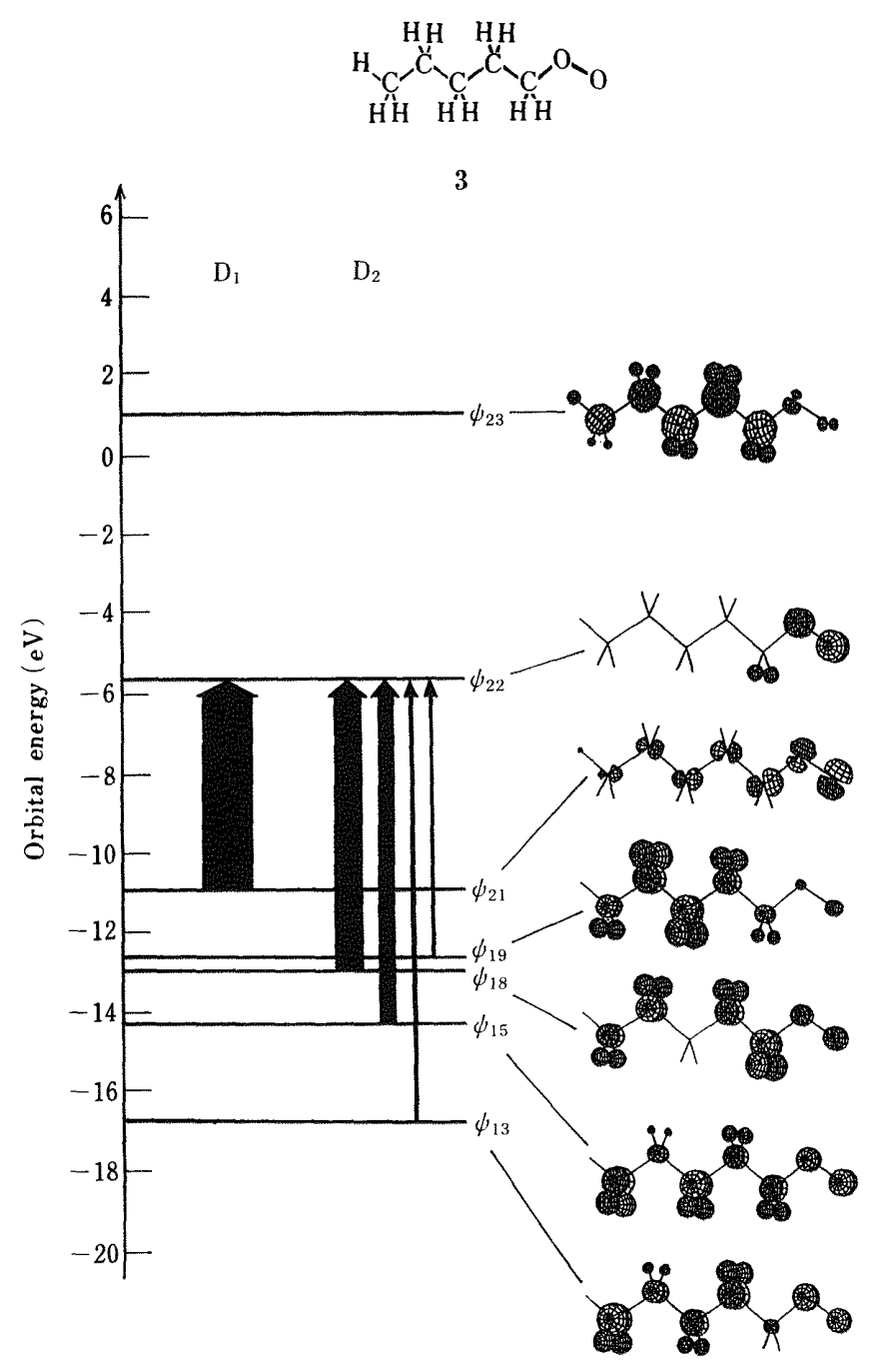

Fig. 3. Configuration Mixing in the $D_{1}$ and $D_{2}$ States and the Relevant Molecular Orbitals for 3
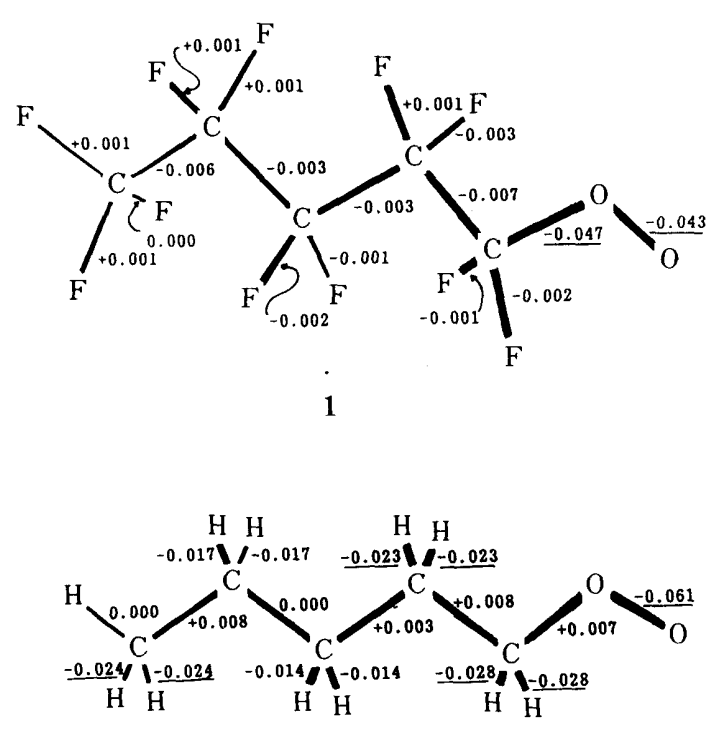

3

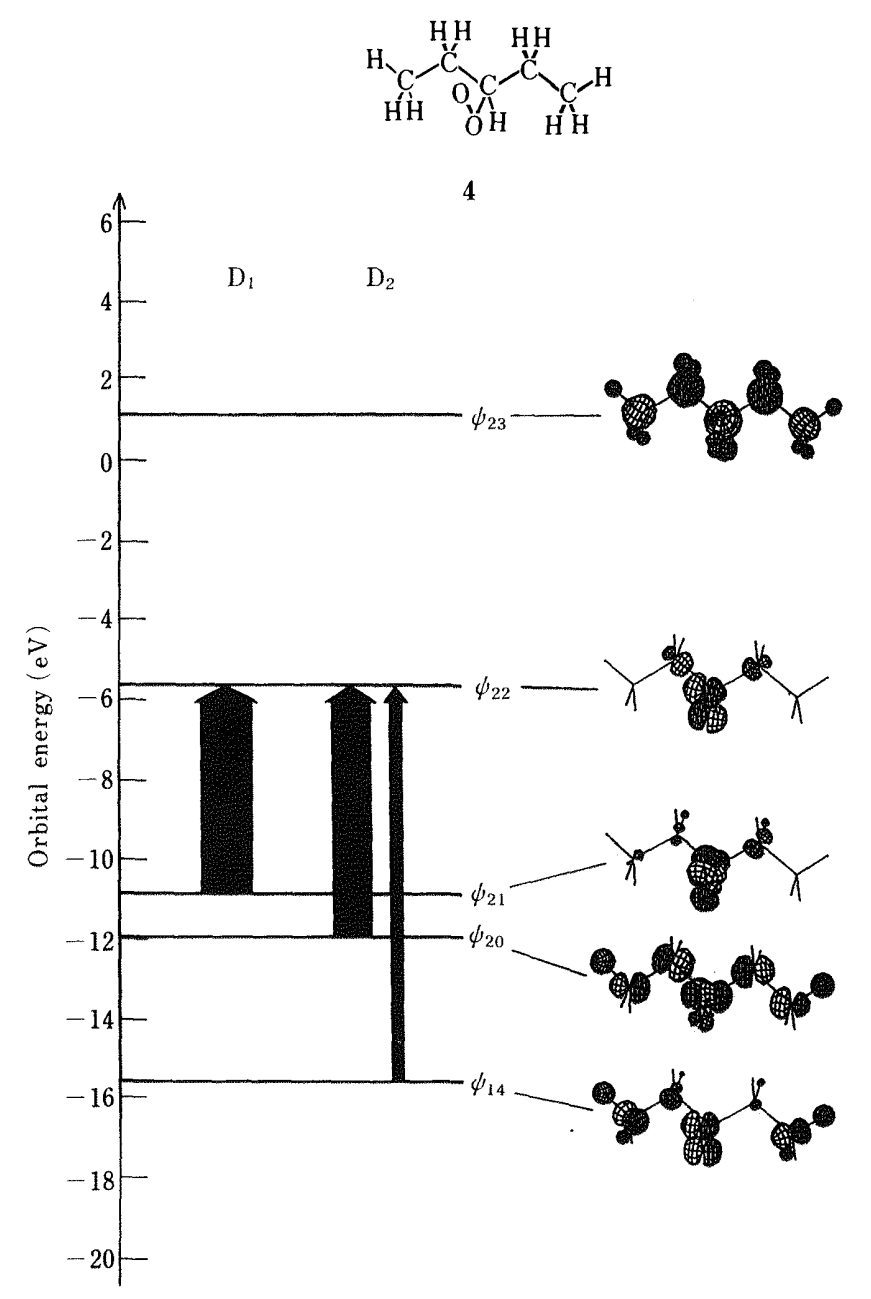

Fig. 4. Configuration Mixing in the $D_{1}$ and $D_{2}$ States and the Relevant Molecular Orbitals for 4 
order and noticeable decreases in the $\mathrm{C}-\mathrm{H}$ bond order.

Although the decrease in the $\mathrm{C}-\mathrm{O}$ bond order on the $\mathrm{D}_{0} \rightarrow \mathrm{D}_{2}$ transition in $\mathbf{1}$ is nearly the same as that in the $\mathrm{O}-\mathrm{O}$ bond order, the $\mathrm{C}-\mathrm{O}$ bond cleavage which results in the formations of alkyl radical and molecular oxygen is considered to be energetically more favorable than the $\mathrm{O}-\mathrm{O}$ bond cleavage which results in the formations of alkyloxy radical and atomic oxygen. On the other hand, the $\mathrm{D}_{0} \rightarrow \mathrm{D}_{2}$ transition in 2 may lead to the $\mathrm{O}-\mathrm{O}$ bond cleavage and the ensuing reaction. It has been reported that the photoirradiation for peroxy radicals of PTFE gives some fragments such as difluoro ketone and carbon monoxide together with endchain perfluoro alkyl radicals. ${ }^{3)}$ The formations of those fragments could be ascribed to the $\mathrm{O}-\mathrm{O}$ bond and $\beta-\mathrm{C}-\mathrm{C}$ bond clevages and the subsequent reactions by atomic oxygen in the midchain peroxy radicals.

For alkyldioxy radicals, 3 and 4, the bond order perturbations on the $\mathrm{D}_{0} \rightarrow \mathrm{D}_{2}$ transition were similar to each other and so the photoreactions may be due to the $\mathrm{O}-\mathrm{O}$ bond cleavage in both types of peroxy radicals.

In conclusion, the MO calculations showed that the photoreaction of peroxy radicals of PTFE is due to the reaction of the midchain peroxy radical in the $D_{2}$ state, based on the calculated transition energies and the oscillator strengths for the $\mathrm{D}_{0} \rightarrow \mathrm{D}_{2}$ transition. Also, the bond order perturbation accompanied by the $\mathrm{D}_{0} \rightarrow \mathrm{D}_{2}$ transition seems to well explain the experimentally known photoreactions of peroxy radicals of PTFE.

\section{Calculation}

The geometries of examined peroxy radicals $(\mathbf{1}, \mathbf{2}, 3$ and 4) were optimized by the AM1-UHF method. ${ }^{6)}$ In the geometry optimization, the conformation of the main chain was fixed in the $15 / 7$ helical structure (the CCCC torsional angle of $166.15^{\circ}$ ), which is experimentally known as the stable structure of PTFE, ${ }^{7)}$ for perfluoro compounds (1 and 2) and in the trans-planar conformation for 3 and
4. The AM1 calculations did not give the known geometries of peroxy radicals and the calculated $\mathrm{C}-\mathrm{O}$ and $\mathrm{O}-\mathrm{O}$ bond lengths were too long and too short, respectively, compared to the known values. ${ }^{8)}$ So, the geometric parameters for the peroxy moiety were taken from the results of the $a b$ initio calculations reported for alkyldioxy radicals. $\left.{ }^{8}\right)$

The molecular orbital calculations for the excited states were performed by the INDO/S-CI method, in which the method and parameters for saturated molecules by Salahub $^{5)}$ were used. The value of the resonance integral between oxygen atoms, $\beta_{00}$, was estimated as $-27.5 \mathrm{eV}$ so as to reproduce the observed absorption wavelength $(255 \mathrm{~nm})$ of methyl peroxy radical. $\left.{ }^{9}\right)$

Calculations were carried out on a FACOM M-780/20 computer at the Computation Center of Nagoya University and on a HITAC M680-H computer at the Computer Center of the Institute of Molecular Science.

Acknowledgement This work was supported in part by a Scientific Research Grant from the Ministry of Education, Science and Culture of Japan (Grant No. 05237228), which is gratefully acknowledged.

\section{References}

1) M. Kuzuya, H. Ito, S. Kondo, N. Noda, A. Noguchi, Macromolecules, 24, 6612 (1991).

2) M. Kuzuya, S. Kondo, H. Ito, A. Noguchi, Appl. Surf. Sci., 60/61, 416 (1922).

3) S. Siegel, H. Hedgpeth, J. Chem. Phys., 46, 3904 (1967).

4) P. Hedvig, J. Polym. Sci., A7, 1145 (1969).

5) D. R. Salahub, Theor. Chim. Acta, 22, 325 (1971); W. Haque, INDOCI/RINDO, Program 372, Quantum Chemistry Program Exchange, University of Indiana, Bloomingston, U.S.A.

6) M. J. S. Dewar, E. G. Zoebisch, E. F. Healy, J. J. P. Stewart, J. Am. Chem. Soc., 107, 3902 (1985); J. J. P. Stewart, MOPAC Ver. 4.0, Program 549, Quantum Chemistry Program Exchange, University of Indiana, Bloomingston, U.S.A.

7) M. Iwasaki, J. Polym. Sci., A1, 1099 (1963).

8) B. H. Besler, M. D. Sevilla, P. MacNeille, J. Phys. Chem., 90, 6446 (1986).

9) M. R. Whitbeck, Diss. Abstr. Int. B, 38, 2204 (1977). 